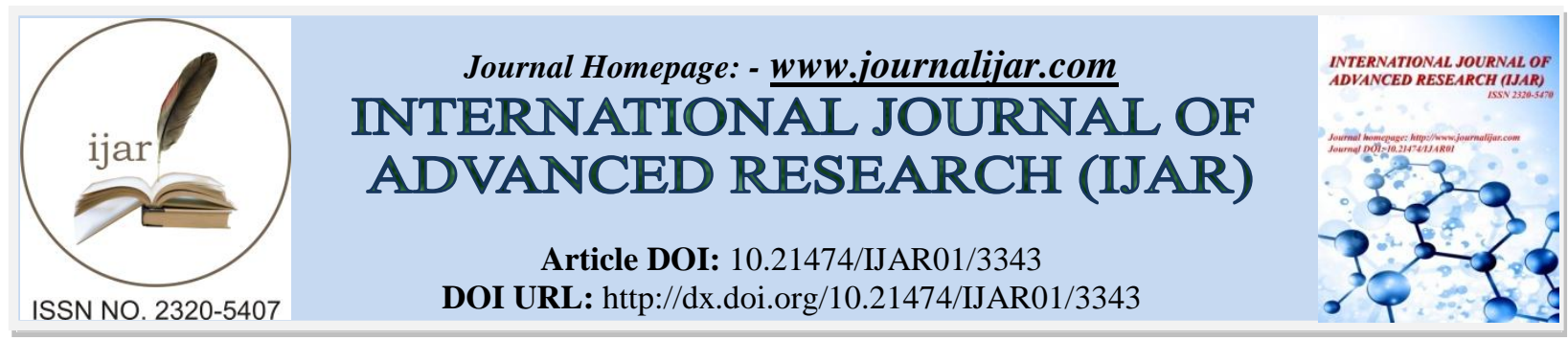

RESEARCH ARTICLE

\title{
A COMPARATIVE STUDY ON KNOWLEDGE REGARDING NEWBORN CARE BETWEEN PRIMI AND MULTI PARA
}

P. Vadivukkarasi Ramanadin ${ }^{1}$ and Manpreet Kaur Bhangu ${ }^{2}$.

1. Professor, Dept. of OBG (N), Shri Vinoba Bhave College of Nursing, Silvassa.

2. M. Sc. Nursing II Year, Mata Sahib Kaur College of Nursing, Mohali.

\section{Manuscript Info}

Manuscript History

Received: 25 December 2016

Final Accepted: 24 January 2017

Published: February 2017

Key words:-

Primipara, Multipara, New born care,

Karl pearson correlation coefficient .

\begin{abstract}
The care a newborn receives depends a lot on the knowledge, skills and attitude of the mother. This study aimed to assess the level of knowledge on new born care between primipara and multipara in selected hospitals of Tricity. A quantitative approach with descriptive, comparative research design was adopted. By purposive sampling technique 160 postnatal mothers were selected. Structured Interview Schedule was used to collect the data from postnatal mothers in civil hospital in the month of December. Study finding shows majority of the primipara mothers $(96.2 \%)$ had average knowledge, $73.8 \%$ of multipara had good knowledge on newborn care.
\end{abstract}

Copy Right, IJAR, 2017,. All rights reserved.

\section{Introduction:-}

The birth of a baby is one of life's most wondrous moments. The first week of life is the most crucial period in the life of an infant. In India 50-60\% of all infant deaths occur within the first month of life. Of these, more than half may die during the first week of birth. This is because the newborn has to adopt itself rapidly and successfully to an alien external environment. The risk of death is greatest during the first 24-48 hrs (PARK K 2005). The ideal basic needs for any newborn include breathing, warmth, cleanliness and mother's milk. Colostrum, the yellowish, sticky breast milk produced at the end of pregnancy, is recommended by WHO as the perfect food for the newborn and feeding should be initiated within one hour of birth (WHO) (UNICEF). Recent researches are concerned with topics of newborn care giving, maternal knowledge and practices because it facilitates growth and development, protection against infection and detection of any abnormalities. The study revealed that mothers' knowledge and practices were within good and satisfactory average scores in most of the studied items related to newborn care giving at home except breast feeding. Significant differences were found between primipara and multipara mothers for most of the studied topics related to different topics of newborn care giving (McMillan J S). The mother is the key person in maintaining the health status of her child.

\section{Materials and Methods:-}

A quantitative approach with descriptive, comparative research design was adopted. The study was conducted in Civil hospital, phase 6 and Liberty hospital, 3b2 Mohali. Sample of the study includes 80 primipara and 80 multipara within 7 days of their delivery who fulfills the inclusion criteria. By using non - probability, purposive sampling technique 160 antenatal mothers were got selected for the present study. Interview schedule with Structured Knowledge Questionnaire on Newborn care was used to collect the data.

Corresponding Author:- P. Vadivukkarasi Ramanadin.

Address:- Professor, Dept. of OBG (N), Shri Vinoba Bhave College of Nursing, Silvassa. 
Results and Discussion:-

Table-1:- Comparison on Level of Knowledge regarding Newborn Care Between Primipara And Multipara $\mathbf{N}=\mathbf{1 6 0}$

\begin{tabular}{|c|c|c|c|c|c|}
\hline \multirow[b]{2}{*}{ S.NO } & \multirow[b]{2}{*}{ Group } & \multicolumn{3}{|c|}{ Level of knowledge on new born care (Score) } & \multirow[b]{2}{*}{$\lambda^{2}, \mathbf{p}$-value } \\
\hline & & $\begin{array}{l}\text { Poor } \\
(0-5)\end{array}$ & $\begin{array}{c}\text { Average } \\
(6-15)\end{array}$ & $\begin{array}{c}\text { Good } \\
(16-20)\end{array}$ & \\
\hline 1 & Primipara & $2.5 \%$ & $96.2 \%$ & $1.2 \%$ & \multirow{2}{*}{$90.067,0.000^{* * *}$} \\
\hline 2 & Multipara & $0 \%$ & $26.2 \%$ & $\mathbf{7 3 . 8 \%}$ & \\
\hline
\end{tabular}

*** Significant

Table-2:- Comparison on Level of Knowledge regarding Aspects of New Born Care Between Primipara \& Multipara

\begin{tabular}{|c|c|c|c|c|c|c|}
\hline \multirow{3}{*}{ Sl.No } & \multirow{3}{*}{ Aspects of Newborn care } & & & & & $\mathrm{N}=160$ \\
\hline & & \multicolumn{2}{|c|}{$\begin{array}{c}\text { Primipara } \\
(\mathbf{N}=\mathbf{8 0})\end{array}$} & \multicolumn{2}{|c|}{$\begin{array}{c}\text { Multipara } \\
(\mathrm{N}=\mathbf{8 0})\end{array}$} & \multirow[t]{2}{*}{ t, p-value } \\
\hline & & Mean & SD & Mean & SD & \\
\hline 1 & Breast feeding & 4.6 & 1.5 & 6.9 & 1.3 & $-10.680,0.000 * * *$ \\
\hline 2 & Eye care & 2.0 & 1.1 & 3.2 & 0.9 & $-7.707,0.000 * * *$ \\
\hline 3 & Thermoregulation & 1.3 & 0.8 & 2.3 & 0.8 & $-8.494,0.000^{* * *}$ \\
\hline 4 & Immunization & 2.6 & 1.2 & 4.1 & 0.9 & $-9.336,0.000 * * *$ \\
\hline 5 & Knowledge score & 10.4 & 2.5 & 16.6 & 2.3 & $-16.645,0.000^{* * * *}$ \\
\hline
\end{tabular}

$$
\text { *** Significant }
$$

Table-3: Association between Knowledge on New Born Care and selected Socio - Demographic Variables among Postnatal Mothers

\begin{tabular}{|c|c|c|c|c|c|}
\hline \multirow{3}{*}{ Sl.No } & \multirow{3}{*}{ Socio - Demographic variables } & & & & $N=160$ \\
\hline & & \multicolumn{3}{|c|}{ Level of knowledge (f) } & \multirow{2}{*}{$\chi^{2}$, df /p value } \\
\hline & & Poor & Average & Good & \\
\hline \multirow[t]{4}{*}{1.} & Age(in years) & & & & \multirow{4}{*}{$\begin{array}{l}5.512,4 / \\
0.238^{\mathrm{NS}}\end{array}$} \\
\hline & $22-24$ & 0 & 36 & 15 & \\
\hline & 25-27 & 1 & 36 & 32 & \\
\hline & $28-30$ & 1 & 26 & 13 & \\
\hline \multirow[t]{6}{*}{2.} & Educational status & & & & \multirow{6}{*}{$\begin{array}{c}11.295,8 / \\
0.185^{\mathrm{NS}}\end{array}$} \\
\hline & Non-formal education & 1 & 16 & 5 & \\
\hline & Primary & 0 & 23 & 21 & \\
\hline & Matric & 1 & 43 & 30 & \\
\hline & Higher secondary & 0 & 14 & 2 & \\
\hline & Graduation & 0 & 2 & 2 & \\
\hline \multirow[t]{4}{*}{3.} & Occupation & & & & \multirow{4}{*}{$\begin{array}{c}8.12,4 / \\
0.0872^{\mathrm{NS}}\end{array}$} \\
\hline & Home maker & 2 & 64 & 51 & \\
\hline & Govt. employee & - & 3 & 1 & \\
\hline & Private employee & - & 31 & 8 & \\
\hline \multirow[t]{6}{*}{4.} & Monthly family income (in Rs.) & & & & \multirow{6}{*}{$\begin{array}{c}8.495,8 / \\
0.386^{\mathrm{NS}}\end{array}$} \\
\hline & $<5000$ & 2 & 26 & 11 & \\
\hline & $5001-10000$ & 0 & 24 & 20 & \\
\hline & 10001-15000 & $\mathbf{0}$ & 33 & 21 & \\
\hline & $15001-20000$ & 0 & 11 & 6 & \\
\hline & $>20000$ & 0 & 4 & 2 & \\
\hline \multirow[t]{4}{*}{5.} & Religion & & & & \multirow{4}{*}{$\begin{array}{c}29.11,4 / \\
0.00000743^{* *}\end{array}$} \\
\hline & Hindu & 1 & 32 & 29 & \\
\hline & Muslim & 0 & 3 & 15 & \\
\hline & Sikh & 1 & 63 & 16 & \\
\hline \multirow[t]{2}{*}{6.} & Type of family & & & & \multirow[b]{2}{*}{$0.02,2 /$} \\
\hline & Joint & 1 & 45 & 28 & \\
\hline
\end{tabular}




\begin{tabular}{|c|c|c|c|c|c|}
\hline & Nuclear & 1 & 53 & 32 & $0.9900^{\mathrm{NS}}$ \\
\hline \multirow[t]{4}{*}{7.} & Area Of residence & & & & \multirow{4}{*}{$\begin{array}{c}76.354,4 / \\
0.000 * *\end{array}$} \\
\hline & Rural & 1 & 29 & 27 & \\
\hline & Semi-urban & 13 & 11 & 0 & \\
\hline & Urban & 1 & 56 & 22 & \\
\hline \multirow[t]{4}{*}{8} & Mode of delivery & & & & \multirow{4}{*}{$\begin{array}{c}19.887,4 / \\
0.00052571^{* *}\end{array}$} \\
\hline & Caesarean & 0 & 17 & 1 & \\
\hline & Vaginal delivery without episiotomy & 18 & $\mathbf{5 0}$ & 3 & \\
\hline & Vaginal delivery with episiotomy & 2 & 63 & 6 & \\
\hline \multirow[t]{4}{*}{9} & Source of information on newborn care & & & & \multirow{4}{*}{$\begin{array}{c}99.046,4 / \\
0.000 * *\end{array}$} \\
\hline & Family members/relatives & 2 & 58 & 23 & \\
\hline & Friends/Peer group & 31 & 0 & 23 & \\
\hline & Mass media & 9 & 0 & 14 & \\
\hline
\end{tabular}

** Significant

Present study shows that majority of the primipara mothers $(96.2 \%)$ had average knowledge, $2.5 \%$ had poor knowledge \& only $1.2 \%$ had good knowledge, whereas most of multipara $(73.8 \%)$ had good \& $26.2 \%$ had average and none them had poor knowledge on newborn care. Calculated chi-square test $\left(\lambda^{2}=\mathbf{9 0 . 0 6 7 , 0 , 0 0 0 , p}<0.05\right)$ shows that multipara have more knowledge on newborn care than the primipara mothers. The supportive study on breast feeding shows that the average knowledge scores was $70.90 \%$.

Present study also shows that the multipara have higher knowledge on breast feeding $(\mathbf{t}=\mathbf{- 1 0 . 6 8 0}, \mathbf{0 . 0 0 0} ; \mathbf{p}<\mathbf{0 . 0 5})$, eye care ( $t=-\mathbf{7 . 7 0 7}, \mathbf{0 . 0 0 0} ; \mathbf{p}<\mathbf{0 . 0 5})$, thermoregulation $(\mathbf{t}=\mathbf{- 8 . 4 9 4 , 0 . 0 0 0 ;} \mathbf{p}<\mathbf{0 . 0 5})$, immunization $(\mathbf{t}=\mathbf{- 9 . 3 3 6}$ $\mathbf{0 . 0 0 0 ;} \mathbf{p}<\mathbf{0 . 0 5}$ ) than the primipara. One more supportive study to assess the level of knowledge of postnatal mother on new born care where as Primipara mothers had comparatively more knowledge on newborn health (mean $\%$ score: 72.13 ) than multipara mothers (mean \% score: 7.38 ).

Present study findings shows that there is an association between the level of knowledge and religion, mode of delivery and source of information. The supported study shows that there was statistically significantly association between knowledge of newborn care with socio demographic variable (3.92) and there was no association found between knowledge score when compared to age, occupation, type of family and religion.

\section{Reference:-}

1. McMillan J S. (2008). October 16. https://www.healthychild.com/care-of-the-newborn/

2. http://www.who.int/topics/breastfeeding/en/reviewed on 15/4/2013.

3. http://www.unicef.org/programme/breastfeeding/baby.htm\#10/.

4. Park K. (2005). Preventive and Social Medicine. $18^{\text {th }}$ Edition. Jabalpur. 391. 